\title{
AUTOMORPHISMS OF THE SYMPLECTIC MODULAR GROUP
}

\author{
BY \\ IRVING REINER
}

1. Introduction. Let $\Omega_{n}$ denote the unimodular group consisting of all $n \times n$ integral matrices of determinant \pm 1 , and let $I^{(n)}$ be the identity matrix in $\Omega_{n}$. We shall use 0 to denote a null matrix whose size is determined by the context, $X^{\prime}$ for the tranpose of $X$, and $X+Y$ for the direct sum of $X$ and $Y$. We call an integral matrix primitive if the greatest common divisor of its maximal size minors is 1 .

\section{Define}

$$
\mathfrak{F}=\left(\begin{array}{cc}
0 & I^{(n)} \\
-I^{(n)} & 0
\end{array}\right),
$$

and let the symplectic group $S p_{2 n}$ consist of all rational $2 n \times 2 n$ matrices $\mathfrak{M}$ satisfÿing

$$
\mathfrak{M F M} \mathfrak{M}^{\prime}=\mathfrak{F}
$$

We define the symplectic modular group $\Gamma_{2 n}$ to be the group of integral matrices in $S p_{2 n}$. Although we shall not do so in this paper, it is sometimes more convenient to work with the factor group of $\Gamma_{2 n}$ over its center $\pm \Im$; see $[1 ; 2 ; 3]\left({ }^{1}\right)$. We may also define an extended group $\Delta_{2 n}$ consisting of all integral matrices $\mathfrak{M}$ for which $\mathfrak{M} \mathfrak{F} \mathfrak{M}^{\prime}= \pm \mathfrak{F}$.

The automorphisms of $S p_{2 n}$ (over any field) have previously been determined [5], as have the automorphisms of $\Gamma_{2}$ (see [4]). The object of this paper is to determine all automorphisms of $\Gamma_{2 n}$. Let us call a homomorphism of $\Gamma_{2 n}$ into $\{ \pm 1\}$ a character. Then we shall prove that every automorphism $\tau$ of $\Gamma_{2 n}$ is given by

$$
\mathfrak{X}^{r}=\psi(\mathfrak{X}) \mathfrak{A X} \mathfrak{X}^{-1} \quad \text { for all } \mathfrak{X} \in \Gamma_{2 n} \text {, }
$$

where $\psi$ is a character, and $\mathscr{U} \in \Delta_{2 n}$. We may remark at this point that the mapping $\sigma$ defined by

$$
\mathfrak{X}^{\sigma}=\mathfrak{X}^{\prime-1} \quad \text { for all } \mathfrak{X} \in \Gamma_{2 n}
$$

is obviously an automorphism. As we shall see, however, it is an inner automorphism.

\section{Let us set}

Presented to the Society, December 28, 1954; received by the editors November 26, 1954.

(1) Numbers in brackets refer to the bibliography at the end of this paper. 


$$
\mathfrak{M}=\left(\begin{array}{ll}
A & B \\
C & D
\end{array}\right)
$$

where $A, B, C, D$ are integral $n \times n$ matrices. Then $\mathfrak{M} \in \Gamma_{2 n}$ if and only if the following conditions are satisfied:

$$
A B^{\prime} \text { symmetric, } C D^{\prime} \text { svmmetric, } A D^{\prime}-B C^{\prime}=I \text {. }
$$

We single out for future use certain types of elements of $\Gamma_{2 n}$ :

(1) Translations:

$$
\mathfrak{T}=\left(\begin{array}{ll}
I & S \\
0 & I
\end{array}\right) \text { or }\left(\begin{array}{ll}
I & 0 \\
S & I
\end{array}\right), \quad S \text { symmetric. }
$$

(2) Rotations:

$$
\Re=\left(\begin{array}{ll}
U & 0 \\
0 & U^{\prime-1}
\end{array}\right)
$$

(3) Semi-involutions:

$$
\subseteq=\left(\begin{array}{cc}
J & I-J \\
J-I & J
\end{array}\right), \quad J \text { diagonal with diagonal elements 0's and 1's. }
$$

Further, if $\mathfrak{M}$ given by (3) is in $\Gamma_{2 n}$, then

$$
\mathfrak{M}^{-1}=\left(\begin{array}{rr}
D^{\prime} & -B^{\prime} \\
-C^{\prime} & A^{\prime}
\end{array}\right)
$$

Finally, if

$$
\mathfrak{M}_{i}=\left(\begin{array}{ll}
A_{i} & B_{i} \\
C_{i} & D_{i}
\end{array}\right) \in \Gamma_{2 n_{i}} \quad(i=1,2),
$$

we define the symplectic direct sum $\mathfrak{M}_{1} * \mathfrak{M}_{2} \in \Gamma_{2\left(n_{1}+n_{2}\right)}$ by

$$
\mathfrak{M}_{1} * \mathfrak{M}_{2}=\left[\begin{array}{llll}
A_{1} & 0 & B_{1} & 0 \\
0 & A_{2} & 0 & B_{2} \\
C_{1} & 0 & D_{1} & 0 \\
0 & C_{2} & 0 & D_{2}
\end{array}\right] .
$$

We may remark that as $\mathfrak{R}$ ranges over all elements of $\Gamma_{2 n}$, the matrix $\left[-I^{(n)}+I^{(n)}\right] \mathfrak{M}$ ranges over all elements in $\Delta_{2 n}^{\prime}=\left\{\mathfrak{X} \in \Delta_{2 n}: \mathfrak{X} \in \Gamma_{2 n}\right\}$. Thence $\mathfrak{M}_{i} \in \Delta_{2 n_{i}}^{\prime}(i=1,2)$ implies $\mathfrak{M}_{1} * \mathfrak{M}_{2} \in \Delta_{2}^{\prime}\left(n_{1}+n_{2}\right)$. However, $\mathfrak{M}_{1} \in \Gamma_{2 n_{1}}$ and $\mathfrak{M}_{2} \in \Delta_{2 n_{2}}$ implies $\mathfrak{M}_{1} * \mathfrak{M}_{2} \notin \Delta_{2\left(n_{1}+n_{2}\right)}$.

2. Involutions in $\Gamma_{2 n}$. It is known [4] that as $x, y$, and $z$ range over all non-negative integers such that $2 x+y+z=n$, the matrix 


$$
W(x, y, z)=\left(\begin{array}{rr}
1 & 0 \\
1 & -1
\end{array}\right)+\cdots+\left(\begin{array}{rr}
1 & 0 \\
1 & -1
\end{array}\right)+(-I)^{(y)}+I^{(\varepsilon)}
$$

(where $x 2 \times 2$ blocks occur) gives a complete set of nonconjugate involutions in $\Omega_{n}$. By an $[x, y, z]$ involution in $\Omega_{n}$ we mean any conjugate of $W(x, y, z)$ in $\Omega_{n}$. Now define

$$
\mathfrak{B}(x, y, z)=W(x, y, z)+W^{\prime}(x, y, z) \in \Gamma_{2 n} .
$$

TheOREM 1. The matrices $\mathfrak{B}(x, y, z)$ with $2 x+y+z=n$ give a complete set of nonconjugate involutions in $\Gamma_{2 n}$.

Proof. We use induction on $n$. The result is trivial for $n=1$, so now let $\mathfrak{X}$ be an involution in $\Gamma_{2 n}, n>1$. From $\mathfrak{X}^{2}=I^{(2 n)}$ we conclude that the characteristic roots of $\mathfrak{X}$ are 1 's and -1 's. Let $\epsilon$ be a characteristic root of $\mathfrak{X}$; then there exists a primitive row vector $\mathfrak{x}$ such that $\mathfrak{x} \mathfrak{X}=\epsilon \mathfrak{x}$. We can then find [6] a matrix $\mathfrak{V} \in \Gamma_{2 n}$ whose first row is $\mathfrak{x}$. In that case the first row of $\mathfrak{X}_{1}=\mathfrak{Y} \mathfrak{X Y}{ }^{-1}$ is $(\epsilon 0 \cdots 0)$. Since $\mathfrak{X}_{1}$ is an involution in $\Gamma_{2 n}$, we obtain

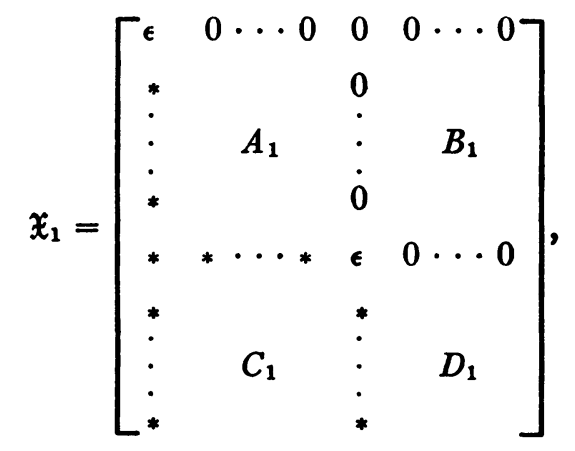

where

$$
\left(\begin{array}{ll}
A_{1} & B_{1} \\
C_{1} & D_{1}
\end{array}\right)
$$

is itself an involution in $\Gamma_{2(n-1)}$. Continuing this procedure, we see that $\mathfrak{X}$ is conjugate in $\Gamma_{2 n}$ to a matrix of the form

$$
\mathfrak{X}_{2}=\left(\begin{array}{ll}
A & 0 \\
C & D
\end{array}\right) \text {. }
$$

From the fact that $\mathfrak{X}_{2}$ is an involution in $\Gamma_{2 n}$, we deduce at once that $A$ is an involution in $\Omega_{n}$, and $D=A^{\prime-1}$. However,

$$
\left(\begin{array}{ll}
U & 0 \\
0 & U^{\prime-1}
\end{array}\right)\left(\begin{array}{ll}
A & 0 \\
C & D
\end{array}\right)\left(\begin{array}{cc}
U^{-1} & 0 \\
0 & U^{\prime}
\end{array}\right)=\left(\begin{array}{cc}
U A U^{-1} & 0 \\
\bar{C} & U^{\prime-1} D U^{\prime}
\end{array}\right)
$$


and so by choosing $U \in \Omega_{n}$ properly, we find that $\mathfrak{X}$ is conjugate to $\mathfrak{X}_{3}$ given by

$$
\mathfrak{X}_{3}=\left(\begin{array}{cc}
W(x, y, z) & 0 \\
C & W^{\prime}(x, y, z)
\end{array}\right)
$$

with a new $C$. Since $\mathfrak{X}_{3} \in \Gamma_{2 n}$ is an involution, we have

$$
C W \text { symmetric, } \quad C \text { skew-symmetric. }
$$

The proof now splits into two cases:

CASE 1. If either $y \neq 0$ or $z \neq 0$, we may set $W(x, y, z)=W_{1} \dot{+}(\epsilon), \epsilon= \pm 1$. From (7) we find that

$$
\mathfrak{X}_{3}=\left[\begin{array}{cccc}
W_{1} & 0 & 0 & 0 \\
0 & \epsilon & 0 & 0 \\
C_{1} & -\mathfrak{x}^{\prime} & W_{1}^{\prime} & 0 \\
\mathfrak{x} & 0 & 0 & \epsilon
\end{array}\right]
$$

and that

$$
\mathfrak{Z}=\left(\begin{array}{ll}
W_{1} & 0 \\
C_{1} & W_{1}^{\prime}
\end{array}\right)
$$

is an involution in $\Gamma_{2(n-1)}$. By the induction hypothesis there exist integers $x_{1}, y_{1}, z_{1}$ with $2 x_{1}+y_{1}+z_{1}=n-1$, such that 8 is conjugate to $\mathfrak{B}\left(x_{1}, y_{1}, z_{1}\right)$. For the moment set $P=W\left(x_{1}, y_{1}, z_{1}\right)$. Then in $\Gamma_{2 n}, \mathfrak{X}_{3}$ is conjugate to $\mathfrak{X}_{4}$, where

$$
\mathfrak{X}_{4}=\left[\begin{array}{cccc}
P & 0 & 0 & 0 \\
0 & \epsilon & 0 & 0 \\
0 & -\mathfrak{x}^{\prime} & P^{\prime} & 0 \\
\mathfrak{x} & 0 & 0 & \epsilon
\end{array}\right]
$$

with a new $\mathfrak{x}$. But then

$$
\mathfrak{X}_{5}=\Im \mathfrak{X}_{4} \Im^{-1}=\left[\begin{array}{llll}
P & 0 & 0 & 0 \\
\mathfrak{x} & \epsilon & 0 & 0 \\
0 & 0 & P^{\prime} & \mathfrak{x}^{\prime} \\
0 & 0 & 0 & \epsilon
\end{array}\right] \text { where } \subseteq=\left[\begin{array}{cccc}
I^{(n-1)} & 0 & 0 & 0 \\
0 & 0 & 0 & 1 \\
0 & 0 & I^{(n-1)} & 0 \\
0 & -1 & 0 & 0
\end{array}\right] \text {. }
$$

Since $\mathfrak{X}_{b}$ is now a direct sum $W+W^{\prime}$, where $W$ is an involution in $\Omega_{n}$, the result follows upon transforming $\mathfrak{X}_{5}$ by a suitably chosen rotation in $\Gamma_{2 n}$.

CASE 2. If both $y$ and $z$ are 0 , we write $W(x, y, z)=L+W_{1}$, where

$$
L=\left(\begin{array}{rr}
1 & 0 \\
1 & -1
\end{array}\right)
$$


Then, as before, $\mathfrak{X}_{3}$ is conjugate to $\mathfrak{X}_{4}$ given by

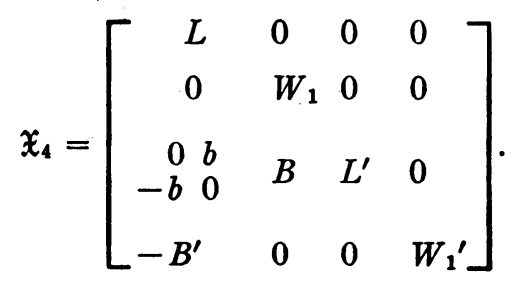

However,

$$
\mathfrak{M}=\left[\begin{array}{cccc}
0 & 0 & I^{(2)} & 0 \\
0 & I^{(n-2)} & 0 & 0 \\
-I^{(2)} & 0 & 0 & 0 \\
0 & 0 & 0 & I^{(n-2)}
\end{array}\right]\left[\begin{array}{cc}
I^{(n)} & 0 \\
& \\
0+b+0^{(n-2)} & I^{(n)}
\end{array}\right] \in \Gamma_{2 n,}
$$

and we have

$$
\mathfrak{M X}_{4} \mathfrak{M}^{-1}=\left(\begin{array}{ll}
L^{\prime} & B \\
0 & W_{1}
\end{array}\right)+\left(\begin{array}{ll}
L & 0 \\
B^{\prime} & W_{1}^{\prime}
\end{array}\right) .
$$

The result then follows as in the previous case.

We have thus shown that any involution $\mathfrak{X} \in \Gamma_{2 n}$ is conjugate to some $\mathfrak{W}(x, y, z)$. On the other hand, if $\mathfrak{B}(x, y, z)$ and $\mathfrak{B}\left(x_{0}, y_{0}, z_{0}\right)$ were conjugate in $\Gamma_{2 n}$, they would certainly be conjugate in $\Omega_{2 n}$. This implies [4] that $x=x_{0}$, $y=y_{0}$, and $z=z_{0}$.

The conjugates of $\mathfrak{B}(x, y, z)$ in $\Gamma_{2 n}$ will be called $(x, y, z)$ involutions.

3. Characterization of the $\pm(0,1, n-1)$ involutions. In $S p_{2 n}$, every involution is conjugate to one of the form $I^{(2 p)} *-I^{(2 q)}$, with $p+q=n$. Any involution in the class of $I^{(2 p)} *-I^{(2 q)}$ is said to have signature $\{p, q\}$ (see [5]). One easily proves that any $(x, y, z)$ involution in $\Gamma_{2 n}$ has signature $\{x+z, x+y\}$, and that the negative of an $(x, y, z)$ involution is of type $(x, z, y)$.

It is known that an abelian set of involutions of signature $\{p, q\}$ in $S p_{2 n}$ cannot contain more than $C_{n, p}$ elements (see [5, Theorem $\left.2 ; 7, \S 19\right]$ ). We shall use this fact in proving the following basic result:

THEOREM 2. Under any automorphism of $\Gamma_{2 n}$, the image of $a(0,1, n-1)$ involution is either $a(0,1, n-1)$ involution or $a(0, n-1,1)$ involution.

Proof. (i) An abelian set of involutions in $\Gamma_{2 n}$, each of type $(x, y, z)$, we shall call an $(x, y, z)$ set. Let $f(x, y, z)$ be the number of elements in an $(x, y, z)$ set of largest size. The above-quoted result shows that

$$
f(x, y, z) \leqq C_{n, x+z},
$$

so for $(x, y, z)= \pm(0,0, n), \pm(0,1, n-1), \pm(1,0, n-2)$ we have $f(x, y, z) \leqq n$. 
We now show that $f(x, y, z)>n$ except for the 6 cases given above.

From an abelian set $\operatorname{xc}$ of $[x, y, z]$ involutions in $\Omega_{n}$, one obtains an $(x, y, z)$ set in $\Gamma_{2 n}$ by taking the set of matrices $U+U^{\prime-1}, U \in J C$. We know, however, that there exist abelian sets of $[x, y, z]$ involutions in $\Omega_{n}$ containing more than $n$ elements, except for the 6 cases listed above (see $[8, \S \S 12$ and 13]).

(ii) The $\pm(0,0, n)$ involutions in $\Gamma_{2 n}$ are $\pm I^{(2 n)}$, so that certainly a $(0,1, n-1)$ involution cannot be mapped onto a $\pm(0,0, n)$ involution by an automorphism of $\Gamma_{2 n}$. It remains to prove that the image cannot be of type $\pm(1,0, n-2)$. To begin with, a simple calculation shows that two rotations $U \dot{+} U^{\prime-1}$ and $V \dot{+} V^{\prime-1}$ are conjugate in $\Gamma_{2 n}$ if and only if $U$ and $V$ are conjugate in $\Omega_{n}$. For $n>2$, there are at least two nonconjugate $[1,0, n-2]$ sets in $\Omega_{n}$, each containing $n$ elements; on the other hand, there is a unique (up to conjugacy) abelian set of $n[0,1, n-1]$ involutions in $\Omega_{n}$ (see $[8, \S 12]$ ). Hence for $n>2$, the image of a $(0,1, n-1)$ involution in $\Gamma_{2 n}$ must be of type $\pm(0,1, n-1)$.

(iii) The case $n=1$ is trivial, and so we are left with $n=2$. Now we have

$$
I^{(2)} *-I^{(2)}=\left(I^{(2)} *\left(\begin{array}{rr}
0 & 1 \\
-1 & 0
\end{array}\right)\right)^{2},
$$

so any $(0,1,1)$ involution in $\Gamma_{4}$ is the square of some element of $\Gamma_{4}$. We show that the $(1,0,0)$ involutions in $\Gamma_{4}$ are not squares. For suppose that

$$
\left(\begin{array}{ll}
A & B \\
C & D
\end{array}\right)^{2}=\left(\begin{array}{ll}
L & 0 \\
0 & L^{\prime}
\end{array}\right), \text { where }\left(\begin{array}{ll}
A & B \\
C & D
\end{array}\right) \in \Gamma_{4} \text { and } L=\left(\begin{array}{rr}
1 & 0 \\
1 & -1
\end{array}\right) \text {. }
$$

From (5) we then have

$$
\left(\begin{array}{ll}
A & B \\
C & D
\end{array}\right)=\left(\begin{array}{ll}
L & 0 \\
0 & L^{\prime}
\end{array}\right)\left(\begin{array}{rr}
D^{\prime} & -B^{\prime} \\
-C^{\prime} & A^{\prime}
\end{array}\right)
$$

This implies that

$$
\left(\begin{array}{ll}
A & B \\
C & D
\end{array}\right)=\left[\begin{array}{cccc}
a & 0 & 0 & 0 \\
(a-d) / 2 & d & 0 & b \\
c & -2 c & a & (a+d) / 2 \\
-2 c & 4 c & 0 & -d
\end{array}\right] .
$$

Using $A D^{\prime}-B C^{\prime}=I$, we find that

$$
-d^{2}-4 b c=1,
$$

whence $d^{2} \equiv-1(\bmod 4)$, since $a, b, c, d$ are integers. This is impossible, and so the theorem is proved.

4. Automorphisms of $\Gamma_{4}$. As is usually the case with determination of 
automorphisms of a group of matrices, the lower the dimension the more difficult the proof. We begin by stating in (i) some earlier results (see [4]) which will be needed.

(i) The group $\Delta_{2}$ coincides with $\Omega_{2}$, and $\Gamma_{2}$ is the subgroup $\Omega_{2}^{+}$consisting of all elements of $\Omega_{2}$ with determinant +1 . For the remainder of this paper we let

$$
S=\left(\begin{array}{rr}
0 & 1 \\
-1 & 0
\end{array}\right), \quad T=\left(\begin{array}{ll}
1 & 1 \\
0 & 1
\end{array}\right)
$$

Then $S$ and $T$ generate $\Gamma_{2}$, and in any relation $S^{m_{1}} T^{n_{1}} S_{m_{2}}^{m_{2}} T^{n_{2}} \ldots=I$ the sum $m_{1}+n_{1}+m_{2}+n_{2}+\cdots$ is always even. Hence the elements $X \in \Gamma_{2}$ can be classified as even or odd according to the parity of the sum of the exponents when $X$ is expressed as a product of powers of $S$ and $T$. The only nontrivial character of $\Gamma_{2}$ is defined by

$$
\epsilon(X)= \begin{cases}1, & X \text { even, } \\ -1, & X \text { odd }\end{cases}
$$

Then every automorphism $\tau$ of $\Gamma_{2}$ is given by

$$
X^{\tau}=\lambda(X) A X A^{-1}
$$

for all $X \in \Gamma_{2}$,

where $\lambda$ is a character, and $A \in \Omega_{2}$.

(ii) Now let $\tau$ be any automorphism of $\Gamma_{4}$. After changing $\tau$ by a suitable inner automorphism, we may assume that $\mathfrak{B}^{r}= \pm \mathfrak{B}$, where

$$
\Re=I^{(2)} *-I^{(2)} \text {. }
$$

Since $\mathfrak{P}$ and $-\mathfrak{B}$ are conjugate in $\Gamma_{4}$, assume in fact that $\mathfrak{B}^{r}=\mathfrak{B}$. Then any element of $\Gamma_{\mathbb{4}}$ which commutes with $\mathfrak{P}$ maps into another such element, so that

$$
\left(Y_{1} * Z_{1}\right)^{r}=Y_{2} * Z_{2}
$$

where $Y_{1}, Y_{2}, Z_{1}, Z_{2} \in \Gamma_{2}$. Let us set

$$
\begin{aligned}
& (Y * I)^{r}=Y^{\alpha} * Y^{\beta} \\
& (I * Z)^{r}=Z^{\gamma} Z^{\delta}
\end{aligned}
$$

for $Y \in \Gamma_{2}$, for $Z \in \Gamma_{2}$.

Then $\alpha, \beta, \gamma, \delta$ are all homomorphisms of $\Gamma_{2}$ into itself, since

$$
\left(Y_{1} * Z_{1}\right)\left(Y_{2} * Z_{2}\right)=Y_{1} Y_{2} * Z_{1} Z_{2}
$$

Further, since $Y * I$ and $I * Z$ commute, so do $Y^{\alpha}$ and $Z^{\gamma}$ for all pairs of elements $Y, Z \in \Gamma_{2}$; also, every element of $\Gamma_{2}$ is a product $Y^{\alpha} Z^{\gamma}$ for some such pair. Since $S \in \Gamma_{2}$, there exists an element $X \in \Gamma_{2}$ such that $S X^{-1} \in \Gamma_{2}^{\alpha}$ and $X \in \Gamma_{2}^{\gamma}$. But then $X$ commutes with $S X^{-1}$, whence $X= \pm I$ or $\pm S$. Therefore either $S \in \Gamma_{2}^{\alpha}$ or $S \in \Gamma_{2}^{\gamma}$. 
Suppose now that $S \in \Gamma_{2}^{\alpha}$; since every element of $\Gamma_{2}^{\gamma}$ commutes with $S$, we see that $\Gamma_{2}^{\gamma} \subset\{ \pm I, \pm S\}$. However, $S \in \Gamma_{2}^{\gamma}$ would imply the finiteness of $\Gamma_{2}^{\alpha}$, whence $\Gamma_{2}=\Gamma_{2}^{\alpha} \Gamma_{2}^{\gamma}$ could not be true. Therefore $\Gamma_{2}^{\gamma} \subset\{ \pm I\}$, and then certainly $\Gamma_{2}^{\alpha}=\Gamma_{2}$. Similarly, one of $\Gamma_{2}^{\beta}, \Gamma_{2}^{\delta}$ is $\Gamma_{2}$, and the other is included in $\{ \pm I\}$.

Now we use the fact that $(-\mathfrak{B})^{r}=-\mathfrak{B}$, that is

$$
(-I * I)^{\tau}=-I * I \text {. }
$$

Therefore $(-I)^{\alpha}=-I$; but if $\Gamma_{2}^{\alpha} \subset\{ \pm I\}$, the fact that $-I=S^{2}$ would imply $(-I)^{\alpha}=I$. Hence $\Gamma_{2}^{\alpha}=\Gamma_{2}, \Gamma_{2}^{\gamma} \subset\{ \pm I\}$, and therefore $\Gamma_{2}^{\beta} \subset\{ \pm I\}, \Gamma_{2}^{\delta}=\Gamma_{2}$.

Next we prove that $\alpha$ is an automorphism; we need merely prove that $Y^{\alpha}=I$ implies $Y=I$. But if $Y^{\alpha}=I$, then $(Y * I)^{r}=I * \pm I$. Since $(I * I)^{\tau}$ $=I * I$ and $(I *-I)^{r}=I *-I$, this implies that $Y=I$. By the same reasoning, $\delta$ is also an automorphism.

(iii) Now define

$$
Y_{1} \circ Y_{2}=\left(\begin{array}{ll}
a_{1} & b_{1} \\
c_{1} & d_{1}
\end{array}\right) \circ\left(\begin{array}{ll}
a_{2} & b_{2} \\
c_{2} & d_{2}
\end{array}\right)=\left[\begin{array}{llll}
0 & a_{1} & 0 & b_{1} \\
a_{2} & 0 & b_{2} & 0 \\
0 & c_{1} & 0 & d_{1} \\
c_{2} & 0 & d_{2} & 0
\end{array}\right] .
$$

Then $Y_{1} \circ Y_{2} \in \Gamma_{4}$ if and only if $Y_{1}, Y_{2} \in \Gamma_{2}$. The elements of $\Gamma_{4}$ which anticommute with $\mathfrak{B}$ are of the form $Y_{1} \circ Y_{2}$, and we have

$$
\begin{aligned}
& (A * B)(C \circ D)=A C \circ B D, \\
& (A \circ B)(C * D)=A D \circ B C, \\
& (A \circ B)(C \circ D)=A D * B C .
\end{aligned}
$$

Suppose now that $(I \circ I)^{r}=U \circ V$. Since $(I \circ I)^{2}=I * I$, we have $(U \circ V)^{2}=U V * V U=I * I$, so $V=U^{-1}$. But now let

$$
\mathfrak{X}^{\sigma}=\left(U^{-1} * I\right) \mathfrak{X} \tau(U * I) .
$$

Then $\mathfrak{B}^{\sigma}=\mathfrak{B}, \sigma$ and $\tau$ differ by an inner automorphism, and $(I \circ I)^{\sigma}=I \circ I$. Changing notation, we henceforth assume $\mathfrak{B}^{r}=\mathfrak{B}$ and $(I \circ I)^{r}=I \circ I$. From

$$
(I \circ I)(Y * Z)(I \circ I)=Z * Y
$$

we deduce

$$
(I \circ I)\left(Z^{\gamma} Y^{\alpha} * Y^{\beta} Z^{\delta}\right)(I \circ I)=Y^{\gamma} Z^{\alpha} * Z^{\beta} Y^{\delta} .
$$

Therefore

$$
Z^{\gamma} Y^{\alpha}=Z^{\beta} Y^{\delta}
$$

for all $Y, Z \in \Gamma_{2}$. Hence $\beta=\gamma, \alpha=\delta$. We have thus shown that for any $Y$, $Z \in \Gamma_{2}$ we have 


$$
(Y * Z)^{r}=\lambda(Z) Y^{\alpha} * \lambda(Y) Z^{\alpha},
$$

where $\lambda$ is a character, and $\alpha$ is an automorphism of $\Gamma_{2}$.

(iv) From the discussion in part (i) of this section, we know that there exists a character $\mu$ and an element $A \in \Delta_{2}$ such that $X^{\alpha}=\mu(X) A X A^{-1}$ for all $X \in \Gamma_{2}$. We remark next that if $\mathfrak{B} \in \Delta_{2 n}$, the map $\phi$ defined by $\mathfrak{X}^{\phi}=\mathfrak{B} \mathfrak{X} \mathfrak{B}^{-1}$ for each $\mathfrak{X} \in \Gamma_{2 n}$ is clearly an automorphism of $\Gamma_{2 n}$. In particular, let us define an automorphism $\sigma$ of $\Gamma_{4}$ by

$$
\mathfrak{X}^{\sigma}=\left(A^{-1} * A^{-1}\right) \mathfrak{X}^{\tau}(A * A)
$$

for all $\mathfrak{X} \in \Gamma_{\mathbf{4}}$.

Calling this new automorphism $\tau$ instead of $\sigma$, we then know that

$$
(Y * Z)^{r}=\lambda(Z) \mu(Y) Y * \lambda(Y) \mu(Z) Z
$$

for each pair $Y, Z \in \Gamma_{2}$, and further that

$$
(I \circ I)^{r}=\left(A^{-1} * A^{-1}\right)(I \circ I)(A * A)=I \circ I .
$$

Thence we have

$$
(Y \circ Z)^{\tau}=(Y * Z)^{\tau}(I \circ I)^{\tau}=\lambda(Z) \mu(Y) Y \circ \lambda(Y) \mu(Z) Z .
$$

(v) We apply the above results to the 4 generators of $\Gamma_{4}$, which are given by (see [3])

$$
\Re_{1}=I \circ I, \quad \Re_{2}=T+T^{\prime-1}, \quad \mathfrak{S}_{0}=S * I, \quad \mathfrak{T}_{0}=T * I
$$

(where $S$ and $T$ are defined by (8)). We have at once

$$
\Re_{1}^{\tau}=\Re_{1}, \quad \mathfrak{S}_{0}^{\tau}= \pm S * \pm I, \quad \mathfrak{T}_{0}= \pm T * \pm I, \quad \mathfrak{S}_{0}^{\tau} \mathfrak{T}_{0}^{\tau}=\mathfrak{S}_{0} \mathfrak{T}_{0},
$$

(the last equation holding because $\mathfrak{S}_{0} \mathfrak{T}_{0}$ is a square).

We use now (and again later) an argument due to Hua [5] to find the possible images $\mathfrak{R}_{2}^{\tau}$. Observe that

$$
\left[\begin{array}{lll}
I^{(2)} & 2 n & 0 \\
& 0 & 0 \\
0 & I^{(2)}
\end{array}\right] \text { and }\left[\begin{array}{lll}
I^{(2)} & 0 & 0 \\
& 0 & 2 m \\
0 & & I^{(2)}
\end{array}\right]
$$

are elements of $\Gamma_{4}$ which are invariant under $\tau$; their product is also invariant. Hence the group of all elements of $\Gamma_{4}$ which commute element-wise with the set of matrices of the form

$$
\left[\begin{array}{lll}
I^{(2)} & \lambda_{1} & 0 \\
& 0 & \lambda_{2} \\
0 & & I^{(2)}
\end{array}\right]
$$

$\lambda_{1}, \lambda_{2}$ even integers,

is mapped onto itself by $\tau$. This group is readily found to consist of all elements of $\Gamma_{4}$ of the form 


$$
\left(\begin{array}{ll}
E & B \\
0 & E
\end{array}\right) \text {, where } E=\left(\begin{array}{rr} 
\pm 1 & 0 \\
0 & \pm 1
\end{array}\right) \text { and } E B^{\prime}=B E .
$$

The squares of these elements are the matrices of $\Gamma_{4}$ given by

$$
\left(\begin{array}{ll}
I & M \\
0 & I
\end{array}\right)
$$

where $M$ is symmetric and all elements of $M$ are even. Hence

$$
\left(\begin{array}{ll}
I & M \\
0 & I
\end{array}\right)^{r}=\left(\begin{array}{ll}
I & M_{1} \\
0 & I
\end{array}\right)
$$

for even symmetric $M$, and $M_{1}$ is also even and symmetric.

Next observe that

$$
\left(\begin{array}{rr}
0 & I \\
-I & 0
\end{array}\right)\left(\begin{array}{ll}
I & M \\
0 & I
\end{array}\right)\left(\begin{array}{rr}
0 & -I \\
I & 0
\end{array}\right)=\left(\begin{array}{cc}
I & 0 \\
-M & I
\end{array}\right)
$$

Since

$$
\left(\begin{array}{rr}
0 & \Gamma \\
-I & 0
\end{array}\right)^{\tau}=(S * S)^{\tau}= \pm S * \pm S
$$

we see that for even symmetric $N$ we have

$$
\left(\begin{array}{ll}
I & 0 \\
N & I
\end{array}\right)^{\top}=\left(\begin{array}{ll}
I & 0 \\
N_{1} & I
\end{array}\right)
$$

with $N_{1}$ even and symmetric.

Now let $\Sigma$ be the group of matrices of the form (9) with $M$ even and symmetric, and let $\Sigma^{\prime}$ be the group of matrices given by (10) with even symmetric $N$. Then $\tau$ maps both $\Sigma$ and $\Sigma^{\prime}$ onto themselves, and so any element commuting with both $\Sigma$ and $\Sigma^{\prime}$ maps into another such element. However, these elements are precisely the rotations in $\Gamma_{4}$. Hence for each $U \in \Omega_{2}$ we have

$$
\left(\begin{array}{cc}
U & 0 \\
0 & U^{\prime-1}
\end{array}\right)^{\prime}=\left(\begin{array}{cc}
U^{\sigma} & 0 \\
0 & \left(U^{\sigma}\right)^{\prime-1}
\end{array}\right)
$$

The map $U \rightarrow U^{\circ}$ is an automorphism $\sigma$ of $\Omega_{2}$, and we already know from $\mathfrak{B}^{r}=\mathfrak{B}$ and $\Re_{1}^{r}=\Re_{1}$ that $S^{\sigma}=S$. Consequently (see [4]) there are only 4 possibilities for $T^{\sigma}$, given by

$$
\left(\begin{array}{ll}
1 & 1 \\
0 & 1
\end{array}\right), \quad\left(\begin{array}{rr}
-1 & 1 \\
0 & -1
\end{array}\right), \quad\left(\begin{array}{rr}
1 & 0 \\
-1 & 1
\end{array}\right), \quad\left(\begin{array}{rr}
-1 & 0 \\
-1 & -1
\end{array}\right) .
$$

(vi) We next apply $\tau$ to both sides of the equation 


$$
(S * I)\left(\begin{array}{ll}
T^{2} & 0 \\
0 & T^{\prime-2}
\end{array}\right)(S * I)^{-1}=\left[\begin{array}{rrrl}
1 & 0 & 0 & 0 \\
0 & 1 & 0 & 0 \\
0 & -2 & 1 & 0 \\
-2 & 0 & 0 & 1
\end{array}\right]
$$

and use equation (10). This shows that

$$
\left(T^{2}\right)^{\sigma}=\left(\begin{array}{rr}
1 & \pm 2 \\
0 & 1
\end{array}\right)
$$

and so either

$$
T^{\sigma}=T \text { or } T^{\sigma}=\left(\begin{array}{rr}
1 & 1 \\
0 & -1
\end{array}\right)=T_{1} \text { (say). }
$$

Now we show that $\mathfrak{S}_{0}^{\tau}= \pm \mathfrak{S}_{0}, \mathfrak{T}_{0}^{\tau}= \pm \mathfrak{T}_{0}$. For, $\mathfrak{R}_{2}$ and $\mathfrak{T}_{0}$ commute; hence so do $\Re_{2}^{r}$ and $\mathfrak{I}_{0}^{\tau}$. However, $\Re_{2}^{r}=\Re_{2}$ or $\Re_{2}^{r}=T_{1}+T_{1}^{\prime-1}$, and it is easily verified that $\pm(T *-I)$ does not commute with either of these two possible images of $\Re_{2}$. Therefore $\mathfrak{T}_{0}^{\tau}= \pm(T * I)$, whence $\mathfrak{S}_{0}^{\tau}= \pm(S * I)$.

Next suppose that $\mathfrak{R}_{2}^{\tau}=T_{1}+T_{1}^{\prime-1}$; then define $\tau_{1}$ by $\mathfrak{X}^{\tau_{1}}=\mathfrak{B} \mathfrak{X}^{\tau} \mathfrak{B}^{-1}$. Then $\mathfrak{S}_{0}^{\tau_{1}}=\mathfrak{S}_{0}, \mathfrak{T}_{0}^{\tau_{1}}=\mathfrak{T}_{0}$, and $\mathfrak{R}_{1}^{\tau_{1}}=-\mathfrak{R}_{1}, \Re_{2}^{\tau_{1}}=-\Re_{2}$. We have therefore shown that apart from an "inner" automorphism by an element of $\Delta_{4}$, every automorphism $\tau$ of $\Gamma_{4}$ can be described by

$$
\left(\Re_{1}, \Re_{2}, \mathfrak{S}_{0}, \mathfrak{T}_{0}\right)^{\tau}=\left( \pm \Re_{1}, \pm \Re_{2}, \pm \mathfrak{S}_{0}, \pm \mathfrak{T}_{0}\right),
$$

and the signs must satisfy

$$
\Re_{1}^{\top} \Re_{2}^{\top}=\Re_{1} \Re_{2}, \quad \mathfrak{S}_{0}^{\top} \mathfrak{T}_{0}^{\top}=\mathfrak{S}_{0} \mathfrak{T}_{1}
$$

Thus every automorphism $\tau$ is given by

$$
\mathfrak{X}^{r}=\theta(\mathfrak{X}) \mathfrak{A X Y}-1
$$

for all $\mathfrak{X} \in \Gamma_{4}$,

where $\mathscr{P} \in \Delta_{4}$ and $\theta$ is a character of $\Gamma_{4}$.

(vii) It will be shown in a future note by the author [9] that $\Gamma_{4}$ has exactly one nontrivial character $\theta$, where $\theta$ is the map of $\Gamma_{4}$ into $\{ \pm 1\}$ induced by

$$
\theta\left(\mathfrak{R}_{1}\right)=\theta\left(\mathfrak{R}_{2}\right)=\theta\left(\mathfrak{S}_{0}\right)=\theta\left(\mathfrak{T}_{0}\right)=-1 .
$$

This fact, together with the preceding discussion, settles the question of automorphisms of $\Gamma_{4}$. It will also be shown in the same note that $\Gamma_{2 n}, n>2$, has no nontrivial characters. This result will be needed in finding all automorphisms of $\Gamma_{2 n}$.

5. Automorphisms of $\Gamma_{2 n}, n>2$. We are now ready to prove, by induction on $n$, the following result:

TheOREM 3. For $n>2$, every automorphism $\tau$ of $\Gamma_{2 n}$ is given by 


$$
\mathfrak{X}^{\tau}=\mathfrak{A} \mathfrak{X} \mathfrak{A}^{-1},
$$

where $\mathfrak{A} \in \Delta_{2 n}$ depends only on $\tau$.

Proof. (i) Let $n \geqq 3$; by the induction hypothesis and our previous results, we may assume that every automorphism $\sigma$ of $\Gamma_{2(n-1)}$ is given by

$$
X^{\sigma}=\theta(X) \cdot A X A^{-1} \text {, }
$$

where $A \in \Delta_{2(n-1)}$ and $\theta$ is a character of $\Gamma_{2(n-1)}$. Let $\tau$ be an automorphism of $\Gamma_{2 n}$, and set

$$
\mathfrak{B}=-I^{(2)} * I^{2(n-1)} .
$$

We see from Theorem 2 that after changing $\tau$ by a suitable inner automorphism, we may take $\mathfrak{B}^{r}= \pm \mathfrak{B}$. The elements of $\Gamma_{2 n}$ which commute with $\mathfrak{B}$ are of the form $Y_{1} * Z_{1}, Y_{1} \in \Gamma_{2}, Z_{1} \in \Gamma_{2(n-1)}$, so that we have

$$
\left(Y_{1} * Z_{1}\right)^{r}=Y_{2} * Z_{2} \text {. }
$$

Again we set

$$
\begin{array}{lr}
(Y * I)^{r}=Y^{\alpha} * Y^{\beta} & \text { for } Y \in \Gamma_{2}, \\
(I * Z)^{\tau}=Z^{\gamma} Z^{\delta} & \text { for } Z \in \Gamma_{2(n-1)} .
\end{array}
$$

Then $\Gamma_{2}^{\alpha}$ and $\Gamma_{2(n-1)}^{\gamma}$ commute elementwise, and $\Gamma_{2}$ is their product. As in $\S 4$, part (ii), we deduce that one of $\Gamma_{2}^{\alpha}, \Gamma_{2(n-1)}^{\gamma}$ is $\Gamma_{2}$, and the other is contained in $\{ \pm I\}$.

(ii) For the moment set $\mathcal{A}=\Gamma_{2}^{\beta}, \mathcal{B}=\Gamma_{2(n-1)}^{\delta}$. Then $\mathcal{A}$ and $\mathcal{B}$ commute elementwise, and their product is $\Gamma_{2(n-1)}$. This shows that $B$ is a normal subgroup of $\Gamma_{2(n-1)}$. We shall show that $A \subset\{ \pm I\}, B=\Gamma_{2(n-1)}$, and that $\delta$ is an automorphism.

For each involution $W \in \Gamma_{2(n-1)}$ we have $\left(W^{\delta}\right)^{2}=I^{\delta}=I$. Suppose that $W^{\delta}= \pm I$ for every involution $W \in \Gamma_{2(n-1)}$; since the involutions in $\Gamma_{2(n-1)}$ generate all of $\Gamma_{2(n-1)}$ (this follows readily from [3]), this would mean that $B \subset\{ \pm I\}$, and so $\beta$ would map $\Gamma_{2}$ homomorphically onto $\Gamma_{2(n-1)}$. We may then show that $\beta$ is an isomorphism; for, suppose that $Y^{\beta}=I, Y \neq I$. Then

$$
(Y * I)^{\tau}=Y^{\alpha} * I \text {. }
$$

Since $B \subset\{ \pm I\}$, certainly $\Gamma_{2(n-1)}^{\gamma}$ is not contained in $\{ \pm I\}$, and so $\Gamma_{2}^{\alpha}$ $\subset\{ \pm I\}$, that is, $\alpha$ is a character. Therefore $Y^{\alpha}= \pm I$. But $Y^{\alpha}=I$ is impossible, since then $(Y * I)^{r}=I^{(2 n)}$ and $Y=I$. On the other hand, $Y^{\alpha}=-I$ is impossible, since in that case $(Y * I)^{\tau}=\mathfrak{B}$, so $(Y * I)= \pm \mathfrak{B}$. Therefore we would have $Y=-I$, and this gives a contradiction because $-I=S^{2}$, and $\alpha$ a character, together imply $(-I)^{\alpha}=I$. Therefore $\beta$ is an isomorphism. However, this is itself impossible because $\Gamma_{2}$ has no involutions other than $\pm I$, whereas $\Gamma_{2(n-1)}$ has such involutions for $n>2$.

We conclude from the abnve that there is at least one involution $W \in \Gamma_{2(n-1)}$ 
for which $W^{\delta} \neq \pm I$. However, $\mathbb{B}$ is a normal subgroup of $\Gamma_{2(n-1)}$, and $W^{\delta} \in \mathbb{B}$. Therefore $\mathscr{B}$ contains all of the conjugates of $W^{\delta}$ in $\Gamma_{2(n-1)}$. It is not difficult to see that if $W^{\delta} \neq \pm I$, the only elements of $\Gamma_{2(n-1)}$ which commute elementwise with all conjugates of $W^{\delta}$ are $\pm I$. Hence $A \subset\{ \pm I\}$, and $\mathcal{B}=\Gamma_{2(n-1)}$. Consequently

$$
(Y * Z)^{\tau}=\theta(Z) Y^{\alpha} * \lambda(Y) Z^{\delta},
$$

where $\theta$ and $\lambda$ are characters, $\alpha$ is a homomorphism of $\Gamma_{2}$ onto itself, and $\delta$ a homomorphism of $\Gamma_{2(n-1)}$ onto itself. We deduce readily that $\alpha$ and $\delta$ are automorphisms, whence incidentally $\mathfrak{B}^{r}=\mathfrak{B}$.

By the discussion at the beginning of the proof, we know that there exist matrices $C \in \Omega_{2}, D \notin \Delta_{2(n-1)}$, and characters $\mu, \nu$ such that

$$
Y^{\alpha}=\mu(Y) C Y C^{-1}, \quad Z^{\delta}=\nu(Z) D Z D^{-1} .
$$

If $C * D \in \Delta_{2 n}$, define $\tau_{1}$ by

$$
\mathfrak{X}^{r_{1}}=(C * D)^{-1} \mathfrak{X}^{\tau}(C * D),
$$

so that

$$
(Y * Z)^{r_{1}}=\theta(Z) \mu(Y) Y * \lambda(Y) \nu(Z) Z .
$$

However, possibly $C * D \in \Delta_{2 n}$. In that case, if $K=(-1)+(1)$, then $C K * D$ $\in \Delta_{2 n}$, and we define $\tau_{2}$ by

$$
\mathfrak{X}^{\tau_{2}}=(C K * D)^{-1} \mathfrak{X}(C K * D) .
$$

Thus, changing notation, we may assume that

$$
(Y * Z)^{r}=\theta(Z) \mu(Y) H Y H^{-1} * \lambda(Y) \nu(Z) Z,
$$

for any $Y \in \Gamma_{2}, Z \in \Gamma_{2(n-1)}$, where $\theta, \mu, \lambda, \nu$ are characters, and where either $H=I^{(2)}$ or $H=K$.

(iii) Suppose now that $Y \in \Gamma_{2}, Z \in \Gamma_{2(n-1)}$ are given by

$$
Y=\left(\begin{array}{ll}
a & b \\
c & d
\end{array}\right), \quad Z=\left(\begin{array}{ll}
A & B \\
C & D
\end{array}\right) .
$$

Then define $Y *^{i} Z$ to be the $2 n \times 2 n$ matrix $\mathfrak{M}$ obtained by placing the elements of $Y$ at the intersections of the $i$ th and $(n+i)$ th rows and columns, filling in the remaining places in those rows and columns with 0 's, and letting the matrix obtained from $\mathfrak{R}$ by deleting the $i$ th and $(n+i)$ th rows and columns be identical with $Z$. Then $Y *^{i} Z$ is a generalization of the previously defined symplectic direct sum, and in fact $Y *^{1} Z=Y * Z$.

Now set

$$
\mathfrak{B}_{i}=-I^{(2)}{ }_{*}^{i} I^{2(n-1)}=I^{(2)} * Q_{i} \text {, say. }
$$

Then $Q_{i}$ is a square in $\Gamma_{2(n-1)}$ (since $\left.-I=S^{2}\right)$, and so from (11) we have 


$$
\mathfrak{B}_{i}^{\tau}=I * Q_{i}=\Re_{i} \text {. }
$$

As before it then follows for $Y \in \Gamma_{2}, Z \in \Gamma_{2(n-1)}$ that

$$
\left(Y *^{i} Z\right)^{\tau}=\left(F_{i}(Z) f_{i}(Y) A_{i} Y A_{i}^{-1}\right) *^{i}\left(g_{i}(Y) G_{i}(Z) B_{i} Z B_{i}^{-1}\right),
$$

where $A_{i} \in \Omega_{2}, B_{i} \in \Delta_{2(n-1)}$, and $F_{i}, f_{i}, g_{i}, G_{i}$ are characters.

(iv) Next let $X$ and $Y \in \Gamma_{2}, Z \in \Gamma_{2(n-2)}$. Applying $\tau$ to both sides of the equation

$$
X *(Y * Z)=Y *^{2}(X * Z)
$$

and using (12), we obtain

$$
\begin{aligned}
& {\left[F_{1}(Y * Z) f_{1}(X) A_{1} X A_{1}^{-1}\right] *\left[g_{1}(X) G_{1}(Y * Z) B_{1}(Y * Z) B_{1}^{-1}\right]} \\
& =\left[F_{2}(X * Z) f_{2}(Y) A_{2} Y A_{2}^{-1}\right] *^{2}\left[g_{2}(Y) G_{2}(X * Z) B_{2}(X * Z) B_{2}^{-1}\right] .
\end{aligned}
$$

In particular for $X=-I, Y=I, Z=I$ this yields

$$
B_{2}(-I * I) B_{2}^{-1}=-I * I \text {, }
$$

so that

$$
B_{2}= \pm A_{1} * C_{2}
$$

and further

$$
B_{1}= \pm A_{2} * \pm C_{2}
$$

We use these expressions for $B_{1}$ and $B_{2}$ in (13) and obtain

$$
\begin{aligned}
& F_{1}(Y * Z) f_{1}(X)=g_{2}(Y) G_{2}(X * Z), \\
& F_{2}(X * Z) f_{2}(Y)=g_{1}(X) G_{1}(Y * Z), \\
& g_{1}(X) G_{1}(Y * Z)=g_{2}(Y) G_{2}(X * Z) .
\end{aligned}
$$

These imply that $f_{1}=g_{1}$ and $f_{2}=g_{2}$.

Continuing in this way we see that each $B_{i}$ decomposes completely, and in fact if

$$
\mathfrak{D}=A_{1} * A_{2} * \cdots * A_{n},
$$

then $B_{i}$ is obtained from $\mathfrak{D}$ by deleting $A_{i}$ and possibly changing signs of some of the remaining $A$ 's. Furthermore, if any $A_{i} \in \Delta_{2}^{\prime}$, then every $A_{i} \in \Delta_{2}^{\prime}$, since each $B_{i} \in \Delta_{2(n-1)}$. Therefore $\mathfrak{D} \in \Delta_{2 n}$. After a further inner automorphism of $\Gamma_{2 n}$ by a factor of $\mathfrak{D}^{-1}$, we may assume hereafter that

$$
\left(Y *^{i} Z\right)^{r}=f_{i}(Y)\left[F_{i}(Z) Y *^{i} G_{i}(Z) B_{i} Z B_{i^{-1}}\right]
$$

for $Y \in \Gamma_{2}, Z \in \Gamma_{2(n-1)}$, where $f_{i}, F_{i}$ and $G_{i}$ are characters and each $B_{i}$ is of the form $( \pm I) * \cdots *( \pm I)$, and in fact we may take $B_{1}=I$.

(v) Define 


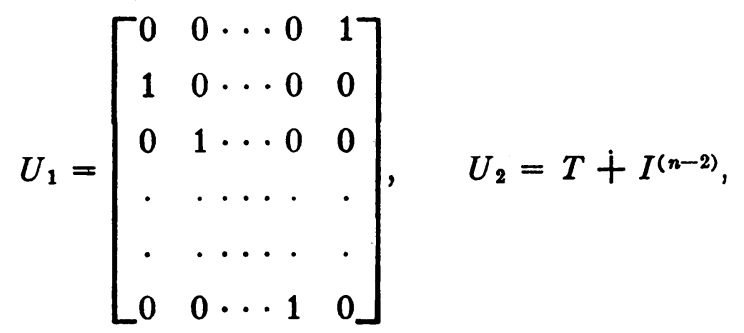

where $T$ is given by (8). Then the generators of $\Gamma_{2 n}$ are (see [3]):

$$
\Re_{1}=U_{1}+U_{1}^{\prime-1}, \quad \Re_{2}=U_{2}+U_{2}^{\prime-1}, \quad \mathfrak{I}_{0}=T * I, \quad \Im_{0}=S * I .
$$

From (14) we find at once that

$$
\mathfrak{T}_{0}^{\tau}= \pm \mathfrak{T}_{0}, \quad \mathfrak{S}_{0}^{\tau}= \pm \mathfrak{S}_{0}, \quad \text { and } \quad \mathfrak{S}_{0}^{\top} \mathfrak{T}_{0}^{\tau}=\mathfrak{S}_{0} \mathfrak{T}_{0}
$$

Next, the rotations of $\Gamma_{2 n}$ map onto rotations under $\tau$, since the rotations are generated by the elements $Y *^{i} Z, i=1, \cdots, n$, where $Y$ and $Z$ have the forms

$$
Y=\left(\begin{array}{ll}
a & 0 \\
0 & d
\end{array}\right), \quad Z=\left(\begin{array}{ll}
A & 0 \\
0 & D
\end{array}\right),
$$

and the image of any such $Y *^{i} Z$ is of the same kind. Therefore $\tau$ induces an automorphism $\sigma$ on the group $\Omega_{n}$, where

$$
\left(\begin{array}{cc}
V & 0 \\
0 & V^{\prime-1}
\end{array}\right)^{\tau}=\left(\begin{array}{cc}
V^{\sigma} & 0 \\
0 & \left(V^{\sigma}\right)^{\prime-1}
\end{array}\right) .
$$

We then know [4] that there exists $H \in \Omega_{n}$ such that

$$
V^{\cdot}=H V^{\omega} H^{-1}
$$

for all $V \in \Omega_{n}$,

where either $V^{\omega}=V$ for all $V$ or $V^{\omega}=V^{\prime-1}$ for all $V$.

We know furthermore that $\tau$ maps every rotation $\mathfrak{P}_{i}$ onto itself, from which we see that $H$ is diagonal, with diagonal elements \pm 1 's. Replace $\tau$ by $\tau_{1}$ defined by

$$
\mathfrak{X}^{r_{1}}=(H+H) \mathfrak{X}^{r}(H+H)
$$

and change notation. We again have $\mathfrak{I}_{0}^{\tau}= \pm \mathfrak{I}_{0}, \mathfrak{S}_{0}^{\tau}= \pm \mathfrak{S}_{0}$, and $\mathfrak{S}_{0}^{\tau} \mathfrak{I}_{0}^{\tau}=\mathfrak{S}_{0} \mathfrak{T}_{0}$, but now $V^{\sigma}=V^{\omega}$ for each $V \in \Omega_{n}$. The argument given in $\$ 4$, parts (iii) and (iv) shows that $\Re_{2}^{\tau}=T^{\prime-1}+T$ is impossible, so $V^{\sigma}=V$ for all $V \in \Omega_{n}$. Therefore $\tau$ is given by

$$
\left(\Re_{1}, \Re_{2}, \mathfrak{T}_{0}, \mathfrak{S}_{0}\right)^{r}=\left(\Re_{1}, \Re_{2}, \pm \mathfrak{I}_{0}, \pm \mathfrak{S}_{0}\right) \text {. }
$$

However, as we have already mentioned, $\Gamma_{2 n}$ has no nontrivial character for $n \geqq 3$. Hence $\mathfrak{T}_{0}^{\tau}=\mathfrak{T}_{0}, \mathfrak{S}_{0}^{\tau}=\mathfrak{S}_{0}$. This completes the proof of the theorem. 
6. We remark finally that if $\mathfrak{M} \in \Gamma_{2 n}$ is given by (3), then

$$
\mathfrak{M}^{\prime-1}=\left(\begin{array}{rr}
D & -C \\
-B & A
\end{array}\right)=\left(\begin{array}{rr}
0 & I \\
-I & 0
\end{array}\right)\left(\begin{array}{ll}
A & B \\
C & D
\end{array}\right)\left(\begin{array}{rr}
0 & -I \\
I & 0
\end{array}\right),
$$

so the automorphism $\sigma: \mathfrak{M}^{\sigma}=\mathfrak{M}^{-1}$ is inner.

Furthermore, any element of $\Delta_{2 n}$ can be written as the product of an element of $\Gamma_{2 n}$ and $-I^{(n)}+I^{(n)}$, so every automorphism of $\Gamma_{2 n}$ can be obtained by using inner automorphisms by elements in $\Gamma_{2 n}$, coupled with the automorphism

$$
\left(\begin{array}{ll}
A & B \\
C & D
\end{array}\right) \rightarrow\left(\begin{array}{rr}
-I & 0 \\
0 & I
\end{array}\right)\left(\begin{array}{ll}
A & B \\
C & D
\end{array}\right)\left(\begin{array}{rr}
-I & 0 \\
0 & I
\end{array}\right)=\left(\begin{array}{rr}
A & -B \\
-C & D
\end{array}\right)
$$

\section{BIBLIOGRAPHY}

1. C. L. Siegel, Math. Ann. vol. 116 (1939) pp. 617-657.

2. L. K. Hua, Trans. Amer. Math. Soc. vol. 57 (1945) pp. 441-490.

3. L. K. Hua and I. Reiner, Trans. Amer. Math. Soc. vol. 65 (1949) pp. 415-426.

4. - Trans. Amer. Math. Soc. vol. 71 (1951) pp. 331-348.

5. L. K. Hua, Ann. of Math. vol. 49 (1948) pp. 739-759.

6. I. Reiner, Trans. Amer. Math. Soc. vol. 77 (1954) pp. 498-505.

7. J. Dieudonné, Memoirs of the American Mathematical Society, No. 2, 1951.

8. I. Reiner, Trans. Amer. Math. Soc. vol. 79 (1955) pp. 459-476.

9. - Real linear characters of the symplectic modular group, to appear in Proc. Amer. Math. Soc.

UNIVERSITY OF ILLINOIS,

URBANA, ILL.

INSTITUTE FOR AdvaNCEd StUdy,

Princeton, N. J. 